九州大学学術情報リポジトリ

Kyushu University Institutional Repository

\title{
Second basic pockets contribute to the localization of PX domains by binding to phosphatidic acid
}

Takeuchi, Hiroshi

Laboratory of Molecular and Cellular Biochemistry, Faculty of Dental Science and Station for Collaborative Research, Kyushu University

\section{Zhang, Zhao}

Laboratory of Molecular and Cellular Biochemistry, Faculty of Dental Science and Station for Collaborative Research, Kyushu University

Gao, Jing

Laboratory of Molecular and Cellular Biochemistry, Faculty of Dental Science and Station for Collaborative Research, Kyushu University

Sugi yama, Goro

Laboratory of Molecular and Cellular Biochemistry, Faculty of Dental Science and Station for Collaborative Research, Kyushu University

他

http://hdl. handle. net/2324/27287

出版情報: Advances in Biological Regulation. 52 (1)，pp.183-194，2012-01. Elsevier バージョン：

権利関係: (C) 2011 Elsevier Ltd. 
Running title: PX domain and phosphatidic acid

\title{
Second basic pockets contribute to the localization of PX domains by binding to phosphatidic acid
}

\author{
Hiroshi Takeuchi, Zhao Zhang, Jing Gao, Goro Sugiyama, Takako Takeuchi, \\ Masato Hirata* \\ Laboratory of Molecular and Cellular Biochemistry, Faculty of Dental Science and Station \\ for Collaborative Research, Kyushu University, Fukuoka 812-8582, Japan
}

Abbreviations: class III PI3K, class III phosphatidylinositol 3-kinase (E.C.2.7.1.137); DGK, diacylglycerol kinase (E.C.2.7.1.107); DMEM, Dulbecco's modified Eagle’s Medium; EEA1, early endosomal antigen 1; ENTH, epsin N-terminal homologue; FYVE, Fab1p/YOTP/Vac1p/EEA1; EGFP, enhanced green fluorescent protein; LPAAT, lysophosphatidic acid acyltransferase (E.C.2.3.1.51); mTOR, mammalian target of rapamycin; PA, phosphatidic acid; PH domain, pleckstrin homology domain; PI(3)P, phosphatidylinositol 3-phosphate; PLC, phospholipase C (E.C.3.1.4.11); PLD, phospholipase D (E.C.3.1.4.4); PMA, phorbol 12-myristate 13-acetate; PX domain, Phox homology domain

*Corresponding author. Tel.: +81-92-642-6317; Fax: +81-92-642-6322.

E-mail address: hirata1@dent.kyushu-u.ac.jp (M. Hirata)

Keywords: PX domain; phosphatidic acid; phosphoinositide; PI3-kinase;

\section{Introduction}

Physiological cellular events, such as growth, cytoskeletal reorganization, membrane trafficking, are mediated by numerous signaling molecules, of which subcellular localizations are regulated strictly. Recruitment of proteins to appropriate sites at correct timings is 
mediated by a number of protein modules whose functions are the interaction with membrane phospholipids (DiNitto et al., 2003). Following the discovery of phosphoinositides as ligands for pleckstrin homology (PH) domains (Harlan et al., 1994; Yagisawa et al., 1994; Garcia et al., 1995; Lemmon et al., 1995; Kanematsu et al., 1996; Takeuchi et al., 1997), numbers of protein domains that mediate protein-lipid interaction have been discovered, including the epsin N-terminal homologue (ENTH), FYVE (Fab1p/YOTP/Vac1p/EEA1) and phox homology (PX) domains (DiNitto et al., 2003). The PX domain is a protein module consisting of approximately 130 amino acids with three $\beta$-strands followed by three $\alpha$-helices forming a helical subdomain, and is found in more than 50 human proteins related to membrane traffic and intracellular signaling (Ellson et al., 2002). Most of the PX domains examined to date specifically recognize phosphatidylinositol 3-phosphate [PI(3)P], which is predominantly found in early endosomes, yet there are some PX domains with binding specificities to different phospholipids (Ellson et al., 2002). Out of the PX domains showing a preference for phospholipids other than PI(3)P, the PX domain of p47phox (p47phox PX) was found to have dual specificity to the phospholipids (Karathanassis et al., 2002; Yaffe, 2002), i.e., there are two distinct pockets on the membrane-binding surface; one prefers $\mathrm{PI}(3,4) \mathrm{P}_{2}$ and the other binds anionic phospholipids such as phosphatidic acid (PA). A series of in vitro experiments showed that simultaneous occupancy of the second pocket by anionic phospholipids may synergistically increases affinity of the PX domain to the membrane containing the phosphoinositide specific for the main pocket (Karathanassis et al., 2002; Stahelin et al., 2003). However, the contribution of the second pocket to spatiotemporal regulation of the PX domain in cells has not been directly investigated in detail.

PA, a membrane phospholipid, is proposed to have intracellular messenger functions to activate a number of physiological events, including secretion, neutrophil superoxide generation, cytoskeletal reorganization and cell growth (English et al., 1996; Ktistakis et al., 2003), but in most cases the defined molecular targets remain to be identified. Three enzymes, phospholipase D (PLD), diacylglycerol kinase (DGK), and lysophosphatidic 
acid acyltransferase (LPAAT), are known to produce PA by hydrolyzing phosphatidylcholine (PC), phosphorylating diacylglycerol (DAG), and adding an acyl chain to lysophosphatidic acid (LPA), respectively. As the amount of PA produced by each enzyme compared to overall PA mass in cells is very little, the enzymes might contribute to cellular signaling by regulating only the localized levels of PA. Although the collection remains small so far, a growing number of putative downstream targets of PA which interact directly with PA have been reported, including the cyclic AMP-specific phosphodiesterase PDE4D3 (Grange et al., 2000), Raf-1 (Ghosh et al., 1996; Rizzo et al., 2000), the mammalian target of rapamycin (mTOR) (Fang et al., 2001), the catalytic subunit of protein phosphatase $1 \gamma$ (Jones and Hannun, 2002; Jones et al., 2005) and Son of sevenless (Zhao et al., 2007). However, a PA-binding module has not yet been reported to date, in contrast to other lipid binding modules such as $\mathrm{PH}$ or FYVE domains for $\mathrm{PI}(4,5) \mathrm{P}_{2}$ or $\mathrm{PI}(3) \mathrm{P}$, respectively.

In the course of examining the specificity of phospholipid-binding of multiple PX domains, we found that the PX domains from two proteins, RPK118 and KIF16B, showed dual specificity to PI(3)P and PA. In the present study, we investigated the role of binding of the PX domains to two different phospholipids, with special reference to the function of these modules as downstream targets of PA.

\section{Materials and Methods}

Materials

Phosphoinositides were obtained from Cell Signals (Lexington, KY) or Echelon Biosciences (Salt Lake City, UT) and other phospholipids were from Avanti Polar Lipids (Alabaster, AL). DGK from Escherichia coli and PLD from Streptomyces chromofuscus were purchased from Sigma-Aldrich (St Louis, MO), and phosphatidylinositol-specific PLC from Bacillus cereus were from BIOMOL (Enzo Life Science, Farmingdale, NY). Antibodies used 
in this study were as follows: anti-HA antibody (HA11; Babco, Richmond, CA), anti-EEA1 (early endosomal antigen1, BD Biosciences, San Jose, CA) and Cy3-anti-mouse antibody (Jackson Immuno Research Laboratories, West Grove, PA). Wortmannin and phorbol 12-myristate 13-acetate (PMA) were from Sigma-Aldrich.

Cell culture, immunofluorescence and live-cell imaging

MDCK and COS-7 cells were obtained from the American Tissue Culture Collection (ATCC) and maintained in Dulbecco's modified Eagle's medium (DMEM) supplemented with $10 \%$ fetal bovine serum. Transient transfection experiments were carried out with lipofectamine PLUS reagents (Invitrogen, Carlsbad, CA) according to the manufacturer's instructions. The procedures for immunofluorescence and live-cell imaging were described previously (Takeuchi et al., 2010).

\section{Protein-lipid overlay assays}

A protein-lipid overlay assay was performed using GST-fusion proteins exactly as described previously (Kanai et al., 2001). GST-tagged recombinant proteins were expressed using a bacterial expression system, and purified with glutathione-Sepharose 4B (GE Healthcare, Buckinghamshire, England) as described by the manufacturer. To examine the binding of the PX domains to enzymatically produced PA from different sources, lipid-blotted membranes were first prepared and incubated in $3 \%$ non-fat bovine serum albumin, and then treated with either PLD or DGK as follows before incubating with GST-PX proteins. For DGK treatment, the membrane was incubated in DGK buffer (50 mM Tris- $\mathrm{HCl}$ at pH 7.5, 100 mM NaCl, 20 mM NaF, 1 mM EDTA, 10 mM MgCl , 1 mM DTT, $0.1 \%$ non-fat bovine serum albumin, $1 \mathrm{mM}$ ATP) with or without $20 \mu \mathrm{g} / \mathrm{ml}$ DGK at $37^{\circ} \mathrm{C}$ for 5 hours with gentle shaking. For PLD treatment, the membrane was incubated in PLD buffer (20 mM 
HEPES-NaOH at pH 7.2, $100 \mathrm{mM} \mathrm{KCl,} 4 \mathrm{mM} \mathrm{NaCl}, 5 \mathrm{mM} \mathrm{MgCl}$, 1 mM CaCl 2,1 \% non-fat bovine serum albumin) with $200 \mathrm{U} / \mathrm{ml}$ PLD or PLC at $37^{\circ} \mathrm{C}$ for 3 hours with gentle shaking.

\section{Preparation of expression constructs and site-directed mutagenesis of the PX domains}

All expression constructs for the PX domains were generated by polymerase chain reaction using human brain cDNAs as the template and the primers with the appropriate restriction enzymatic sites, followed by subcloning into corresponding vectors, i.e., pGEX-4T-3 (GE Healthcare) and pEGFP-N1 (Clontech Laboratories, Mountain View, CA). Mutants carrying one or two substitutions of amino acid residues were produced using QuikChange site-directed mutagenesis kit (Agilent Technologies, Santa Clara, CA). All constructs were fully sequenced to verify their integrity at Beth Israel Deaconess Medical Center DNA Sequencing Core Facility. More details of all constructs are available on request.

\section{Results and Discussion}

PX domain whose binding specificity is to phosphatidic acid

In the present study, we examined the binding specificity to phosphoinositides using multiple samples of PX domains, since the domain was first identified as that of PI(3)P binding and thus it is now widely accepted that the protein bearing the domain exhibits subcellular localization at early endosomes (EE) due to binding specificity to PI(3)P. However, it is still unclear whether all PX domains show specificity to PI(3)P, and whether only the binding to $\mathrm{PI}(3) \mathrm{P}$ is responsible for the subcellular localization. We first discussed with the database [SMART (Simple Modular Architecture Research Tool)] of PX domains that contains more than 50 proteins; we selected $10 \mathrm{PX}$ domains randomly out of the proteins listed in the database and constructed the genes in conjugation with that of GST to express in 
a bacterial expression system. Purified protein samples were assayed by the protein-lipid overlay assay. The membrane sheets were dotted with a variety of phosphoinositides and anionic phospholipids, phosphatidylserine (PS) and PA at serially diluted concentrations. The PX domains of RPK118 and KIF16B, out of the PX domain examined, showed different binding patterns. As shown in Figure 1, the two proteins exhibited broad specificity, but with relative specificity to PA as well as PI(3)P, showing a clear contrast to SNX19 with definite specificity to PI(3)P. To examine the specific binding to PA further, membrane sheets dotted with phosphatidylcholine (PC) were incubated with PLD to produce PA on the sheet. As shown in Figure 2A, the PX domains of RPK118 and KIF16B showed binding to the spot where PC was first dotted, followed by treatment with PLD, but not with PLC. No binding to diacylglycerol (DAG) and lysophosphatidic acid (LPA) was observed. We next examined this further from a different direction; DAG dotted on the sheet was incubated with DGK in the presence of MgATP, where PA must be yielded. The PX domains of RPK118 and KIF16B showed binding to the spot on the sheet (Figure 2B), supporting the notion that the PX domains from RPK118 and KIF16B are capable of binding to PA as well as PI(3)P.

\section{Subcellular localization of the PX domains of RPK118 and KIF16B}

Subcellular localization of the PX domains of RPK118 and KIF16B was observed using MDCK cells following transfection with each gene conjugated with EGFP to monitor under a fluorescent microscope. Both proteins exhibited a dotted appearance near the nucleus, coinciding with EEA1, a marker of early endosomes (Figure 3), indicating that the PX domains whose phosphoinositide binding are specific to PA and PI(3)P also localize at early endosomes.

Importance of the binding to PA was examined in cells treated with wortmannin, causing down-regulation of the cellular PI3K cycle to reduce the subcellular amount of PI(3)P (Volinia et al., 1995; Wurmser et al., 1999). As shown in Figure 4, wortmannin treatment 
markedly reduced the EGFP-positive dotted structures near the nucleus in cells expressing the PX domain of SNX19, which binds very strictly to PI(3)P. The PX domains from RPK118 and KIF16B showed slightly reduced dots by wortmannin treatment, but still the dots remained, indicating that the binding to PA promotes localization at endosomal membranes, along with that to PI(3)P.

Sequence alignment of the PX domain and the amino acid residues responsible for the binding

Figure 5 shows amino acid alignments of several PX domains including RPK118 and KIF16B. 3D structural analysis of p40 phox revealed the region responsible for the binding and the important several basic amino acids which accommodate the phosphate moiety of PI(3)P by forming salt-bridge (Bravo et al., 2001). The mutation of the corresponding arginine (R) at 53 and 1220 in RKP118 and KIF16B, respectively, to glutamine (Q) almost abolished the dotted structures, as shown in Figure 6.

We further examined the effects of mutations at other amino acid residues on lipid binding and subcellular localization using the PX domain of RPK118 (Figure 7). The mutation of R53Q reduced the binding ability to PI(3)P, but that to PA did not appear to be reduced. A similar pattern of binding reduction to PI(3)P was observed with the mutation of tyrosine at 54 to alanine (Y54A). Neither mutation caused specific localization at the endosomal structures as multiple dots; however, the mutation of K52Q did not reduce lipid binding or diminishment of the dotted structures. These results suggest that the binding to PI(3)P of the PX domain from RPK118 is primarily important for localization at endosomal structures.

Next, the effect of mutations at basic amino acids in the region which forms a putative second pocket probably accommodating PA was examined. Only the double mutations at K58Q/K59Q showed a marked reduction in PA binding, showing no dotted structures, indicating that PA binding is also involved in localization at endosomal structures. 


\section{Effect of PLD activation on subcellular localization of the mutant PX domain of RPK118}

To further investigate the contribution of PA binding in determining the subcellular localization of the PX domain, we monitored COS7 cells expressing an EGFP-fused Y54A mutant of the PX domain from RPK118 (RPKPX Y54A), whose binding to PI(3)P was markedly reduced while that to PA remained intact (see Figure 7). Diffuse localization throughout the cytoplasm in cells was observed (Figure 8A; none). The cells were first treated with PMA (100 $\mathrm{nM}$ ) for $10 \mathrm{~min}$ and were expecting to stimulate PLD to increase PA in the cells. PMA treatment caused translocation of RPKPX-Y54A-EGFP to form the dotted structures (Figure 8A). This endosomal localization of RPKPX-Y54A was quickly reduced by the following treatment with wortmannin (Figure 8A). Although wortmannin is known to inhibit ligand-induced PLD activation in the cell, it has been reported that PMA-induced PLD activation was not inhibited by wortmannin (Reinhold et al., 1990; Nakamura et al., 1997), suggesting that the reduced localization of the RPKPX mutant to the dotted structure by wortmannin is probably due to the inhibition of PI3K and subsequent reduction of PI(3)P. As shown in Figure 8B, the dotted structure of RPKPX-Y54A mutant was co-localized with an EEA1-positive structure, thus RPKPX-Y54A translocated to the site where wild-type RPKPX localized in the resting cells (see also Figure 3). As shown in Figure 8C, RPKPX-Y54A was co-localized with exogenously expressed HA-PLD1b on endosomal structures in PMA-treated cells, supporting the notion that the translocation of the mutant to EEA1-positive structures was attributed to PA production on endosomal membranes by PLD. The localization of HA-PLD1b at the Golgi-like structure was consistent with the previous report (Baillie et al., 2002), although PDE4A1 was co-localized with HA-PLD1b not only on the endosomal membrane but also at the Golgi-like structure (Baillie et al., 2002). These data suggested that $\mathrm{PI}(3) \mathrm{P}$ binding is primarily required for endosomal localization of the RPKPX-Y54A mutant, while binding to PA produced by the activation of PLD or DGK 
promotes the subcellular localization.

The role of the two pockets on the surface of the PX domain for recognizing different phospholipids would increase the binding affinity and specificity to the site where the parental molecule localizes in the cell. The reason for having two pockets in one module would be similar to the reason for having multiple modules in one molecule, i.e. multiple modules with different binding partners synergistically function to decide subcellular localization of the parental protein, for example, the FYVE and the second coiled-coil domains for early endosomal localization of Hrs (Raiborg et al., 2001), and the PX and BAR (Bin/Amphiphysin/Rvs) domains for endosomal localization of sorting nexin-1 (Carlton et al., 2004). Indeed, the PX domains from p47 $^{\text {phox }}$ and PLD1, both of which share two basic pockets for phosphoinositide and anionic phospholipid, showed much higher binding affinity to liposomes containing the both phospholipids compared to liposomes containing only a single phospholipid. In agreement with this, the endosomal localization of RPK118 and KIF16B was not sensitive to wortmannin treatment with which the localization of the PI(3)P-specific PX domain was abrogated (Figure 4). However, there would be an additional role of the dual specificity of the PX domains other than increasing the binding affinity to the site, i.e. the PX domains with two different partners can be regulated its spatiotemporal localization by converging two distinct lipid-mediated signaling pathways like a molecule containing two independent modules. The PX domains of RPK118 and KIF16B localized to endosomal membrane where the localizations of both class III PI3K and PLD1 have been shown (Colley et al., 1997; Wurmser et al., 1999; Tuma et al., 2001; Du et al., 2003), suggesting that the localization of those PX domains was controlled by those enzymes whose activities are regulated independently. Disruption of binding to either PI(3)P or PA without affecting binding to the other by substitution of single amino acid (Figure 7) indicated that the affinities of the PX domains to a single lipid, PI(3)P or PA, were not enough to maintain localization, but affinities to both lipids are needed. Thus, the levels of both phospholipids must elevate at the same site and timing. In other words, the PX domains of RPK118 and KIF16B could also 
translocate to the plasma membrane if the levels of both PI(3)P and PA increased at the plasma membrane, e.g. elevation of PI(3)P at the plasma membrane after cellular stimulation by LPA has been reported (Maffucci et al., 2005).

Finally, the finding that the PX domains of RPK118 and KIF16B have a second pocket for accommodating PA, together with previous reports about the existence of a secondary binding site for PA on the PX domains of p47phox and PLD (Karathanassis et al., 2002; Stahelin et al., 2004), would make a subgroup of PX domains the first candidate for a 'universal' PA binding module, which functions as a downstream target of PA.

\section{Summary}

In this study, we discovered that PX domains from RPK118 and KIF16B recognized both PI(3)P and PA. We showed that these PX domains recognized both phospholipids by two distinct pockets analogous to the case of the PX domain of $447^{\text {phox }}$ using a series of point mutants. By using the mutant PX domain, whose binding to PI(3)P was reduced but that to PA remained intact, translocation of the PX domain after activation of PLD was observed, indicating that binding of the second pocket to PA produced by PLD would be important to promote the subcellular localization of the PX domain to the endosomal membrane, where PI(3)P is abundant. For the first time we here showed evidence that PA binding to the second pocket of the PX domain has an important role not only to increase the affinity of the module to a specialized subcellular location, but also to target the module to the site where both of the lipid ligands are localized.

\section{Acknowledgements}

We thank Prof. Mordechai Liscovitch for providing us with the plasmid to express HA-tagged PLD1b. This study was supported by a Grant-in-Aid for Scientific Research from 
the Ministry of Education, Science, Sports, and Culture of Japan (to HT, JG and MH), the Japan China Medical Association (to JG), and the Shimabara Foundation (to HT). ZZ is a graduate student supported by the Japanese Government.

\section{References}

Baillie GS, Huston E, Scotland G, Hodgkin M, Gall I, Peden AH, MacKenzie C, Houslay ES, Currie R, Pettitt TR, Walmsley AR, Wakelam MJ, Warwicker J, Houslay MD. TAPAS-1, a novel microdomain within the unique N-terminal region of the PDE4A1 cAMP-specific phosphodiesterase that allows rapid, Ca2+-triggered membrane association with selectivity for interaction with phosphatidic acid. J Biol Chem 2002;277:28298-309.

Bravo J, Karathanassis D, Pacold CM, Pacold ME, Ellson CD, Anderson KE, Butler PJ, Lavenir I, Perisic O, Hawkins PT, Stephens L, Williams RL. The crystal structure of the PX domain from p40(phox) bound to phosphatidylinositol 3-phosphate. Mol Cell 2001;8:829-39.

Carlton J, Bujny M, Peter BJ, Oorschot VM, Rutherford A, Mellor H, Klumperman J, McMahon HT, Cullen PJ. Sorting nexin-1 mediates tubular endosome-to-TGN transport through coincidence sensing of high- curvature membranes and 3-phosphoinositides. Curr Biol 2004;14:1791-800.

Colley WC, Sung TC, Roll R, Jenco J, Hammond SM, Altshuller Y, Bar-Sagi D, Morris AJ, Frohman MA. Phospholipase D2, a distinct phospholipase D isoform with novel regulatory properties that provokes cytoskeletal reorganization. Curr Biol 1997;7:191-201.

DiNitto JP, Cronin TC, Lambright DG. Membrane recognition and targeting by lipid-binding domains. Sci STKE 2003;2003:re16. 
Du G, Altshuller YM, Vitale N, Huang P, Chasserot-Golaz S, Morris AJ, Bader MF, Frohman MA. Regulation of phospholipase D1 subcellular cycling through coordination of multiple membrane association motifs. J Cell Biol 2003;162:305-15.

Ellson CD, Andrews S, Stephens LR, Hawkins PT. The PX domain: a new phosphoinositide-binding module. J Cell Sci 2002;115:1099-105.

English D, Cui Y, Siddiqui RA. Messenger functions of phosphatidic acid. Chem Phys Lipids 1996;80:117-32.

Fang Y, Vilella-Bach M, Bachmann R, Flanigan A, Chen J. Phosphatidic acid-mediated mitogenic activation of mTOR signaling. Science 2001;294:1942-5.

Garcia P, Gupta R, Shah S, Morris AJ, Rudge SA, Scarlata S, Petrova V, McLaughlin S, Rebecchi MJ. The pleckstrin homology domain of phospholipase C-delta 1 binds with high affinity to phosphatidylinositol 4,5-bisphosphate in bilayer membranes. Biochemistry 1995;34:16228-34.

Ghosh S, Strum JC, Sciorra VA, Daniel L, Bell RM. Raf-1 kinase possesses distinct binding domains for phosphatidylserine and phosphatidic acid. Phosphatidic acid regulates the translocation of Raf-1 in 12-O-tetradecanoylphorbol-13-acetate-stimulated Madin-Darby canine kidney cells. J Biol Chem 1996;271:8472-80.

Grange M, Sette C, Cuomo M, Conti M, Lagarde M, Prigent AF, Nemoz G. The cAMP-specific phosphodiesterase PDE4D3 is regulated by phosphatidic acid binding. Consequences for cAMP signaling pathway and characterization of a phosphatidic acid binding site. J Biol Chem 2000;275:33379-87.

Harlan JE, Hajduk PJ, Yoon HS, Fesik SW. Pleckstrin homology domains bind to phosphatidylinositol-4,5-bisphosphate. Nature 1994;371:168-70. 
Jones JA, Hannun YA. Tight binding inhibition of protein phosphatase-1 by phosphatidic acid. Specificity of inhibition by the phospholipid. J Biol Chem 2002;277:15530-8.

Jones JA, Rawles R, Hannun YA. Identification of a novel phosphatidic acid binding domain in protein phosphatase-1. Biochemistry 2005;44:13235-45.

Kanai F, Liu H, Field SJ, Akbary H, Matsuo T, Brown GE, Cantley LC, Yaffe MB. The PX domains of p47phox and p40phox bind to lipid products of PI(3)K. Nat Cell Biol 2001;3:675-8.

Kanematsu T, Misumi Y, Watanabe Y, Ozaki S, Koga T, Iwanaga S, Ikehara Y, Hirata M. A new inositol 1,4,5-trisphosphate binding protein similar to phospholipase C-delta 1. Biochem J 1996;313:319-25.

Karathanassis D, Stahelin RV, Bravo J, Perisic O, Pacold CM, Cho W, Williams RL. Binding of the PX domain of p47(phox) to phosphatidylinositol 3,4-bisphosphate and phosphatidic acid is masked by an intramolecular interaction. Embo J 2002;21:5057-68.

Ktistakis NT, Delon C, Manifava M, Wood E, Ganley I, Sugars JM. Phospholipase D1 and potential targets of its hydrolysis product, phosphatidic acid. Biochem Soc Trans 2003;31:94-7.

Lemmon MA, Ferguson KM, O'Brien R, Sigler PB, Schlessinger J. Specific and high-affinity binding of inositol phosphates to an isolated pleckstrin homology domain. Proc Natl Acad Sci U S A 1995;92:10472-6.

Maffucci T, Cooke FT, Foster FM, Traer CJ, Fry MJ, Falasca M. Class II phosphoinositide 3-kinase defines a novel signaling pathway in cell migration. $\mathrm{J}$ Cell Biol 2005;169:789-99.

Nakamura M, Nakashima S, Katagiri Y, Nozawa Y. Effect of wortmannin and 
2-(4-morpholinyl)-8-phenyl-4H-1-benzopyran-4-one

(LY294002)

on

$\mathrm{N}$-formyl-methionyl-leucyl-phenylalanine-induced phospholipase $\mathrm{D}$ activation in differentiated HL60 cells: possible involvement of phosphatidylinositol 3-kinase in phospholipase D activation. Biochem Pharmacol 1997;53:1929-36.

Raiborg C, Bremnes B, Mehlum A, Gillooly DJ, D'Arrigo A, Stang E, Stenmark H. FYVE and coiled-coil domains determine the specific localisation of Hrs to early endosomes. $\mathrm{J}$ Cell Sci 2001;114:2255-63.

Reinhold SL, Prescott SM, Zimmerman GA, McIntyre TM. Activation of human neutrophil phospholipase D by three separable mechanisms. Faseb J 1990;4:208-14.

Rizzo MA, Shome K, Watkins SC, Romero G. The recruitment of Raf-1 to membranes is mediated by direct interaction with phosphatidic acid and is independent of association with Ras. J Biol Chem 2000;275:23911-8.

Stahelin RV, Burian A, Bruzik KS, Murray D, Cho W. Membrane binding mechanisms of the PX domains of NADPH oxidase p40phox and p47phox. J Biol Chem 2003;278:14469-79.

Stahelin RV, Ananthanarayanan B, Blatner NR, Singh S, Bruzik KS, Murray D, Cho W. Mechanism of membrane binding of the phospholipase D1 PX domain. J Biol Chem 2004;279:54918-26.

Takeuchi H, Kanematsu T, Misumi Y, Sakane F, Konishi H, Kikkawa U, Watanabe Y, Katan M, Hirata M. Distinct specificity in the binding of inositol phosphates by pleckstrin homology domains of pleckstrin, RAC-protein kinase, diacylglycerol kinase and a new 130 kDa protein. Biochim Biophys Acta 1997;1359:275-85.

Takeuchi H, Takeuchi T, Gao J, Cantley LC, Hirata M. Characterization of PXK as a protein involved in epidermal growth factor receptor trafficking. Mol Cell Biol 
2010;30:1689-702.

Tuma PL, Nyasae LK, Backer JM, Hubbard AL. Vps34p differentially regulates endocytosis from the apical and basolateral domains in polarized hepatic cells. J Cell Biol 2001;154:1197-208.

Volinia S, Dhand R, Vanhaesebroeck B, MacDougall LK, Stein R, Zvelebil MJ, Domin J, Panaretou C, Waterfield MD. A human phosphatidylinositol 3-kinase complex related to the yeast Vps34p-Vps15p protein sorting system. Embo J 1995;14:3339-48.

Wurmser AE, Gary JD, Emr SD. Phosphoinositide 3-kinases and their FYVE domain-containing effectors as regulators of vacuolar/lysosomal membrane trafficking pathways. J Biol Chem 1999;274:9129-32.

Yaffe MB. The p47phox PX domain: two heads are better than one! Structure 2002;10:1288-90.

Yagisawa H, Hirata M, Kanematsu T, Watanabe Y, Ozaki S, Sakuma K, Tanaka H, Yabuta N, Kamata H, Hirata $\mathrm{H}$, et al. Expression and characterization of an inositol 1,4,5-trisphosphate binding domain of phosphatidylinositol-specific phospholipase C-delta 1. J Biol Chem 1994;269:20179-88.

Zhao C, Du G, Skowronek K, Frohman MA, Bar-Sagi D. Phospholipase D2-generated phosphatidic acid couples EGFR stimulation to Ras activation by Sos. Nat Cell Biol 2007;9:706-12. 


\section{Figure legends}

Fig. 1. Specificity of phospholipid binding of the PX domains. Serially diluted phospholipids were dotted on nitrocellulose membranes and probed with isolated PX domains from various proteins prepared as GST-fusion proteins using a bacterial expression system. GST-fused proteins bound to the lipids were detected with anti-GST antibody. GST protein (1 pmol) spotted as a positive control for detection with the antibody is observed in the upper right corner of the membrane probed with GST.

Fig. 2. Binding of the PX domains to PA enzymatically-produced on the membrane. A: Membrane sheets spotted with the serial dilutions of the lipids were incubated with PLD or PLC, and then probed with PX domains from the indicated protein, as in Fig. 1. B: Membrane sheets with dotted lipids were incubated with DGK in the presence of MgATP and subjected to a protein-lipid overlay assay, as described in Fig. 1.

Fig. 3. Subcellular localization of EGFP-fused PX domains in the cells. MDCK cells cultured on glass coverslips were transiently transfected to express EGFP-fused PX domains from RPK118 or KIF16B in the growing medium, and then fixed with PFA and subjected to immunocytochemistry. EGFP-PX domains were visualized in green, EEA1 in red by indirect immunofluorescence using a combination of mouse antibody against EEA1 and Cy3-conjugated anti-mouse IgG, and then the cells were counterstained with 4', 6-diamidino-2-phenylindole (blue). The yellowish staining in the merged picture indicates co-localization of EGFP-PX domains with EEA1. Scale bars, $10 \mu \mathrm{m}$.

Fig. 4. Sensitivity of endosomal localization of the PX domains to PI3K inhibitor. MDCK cells cultured on coverslips were transiently transfected in growing medium to express 
EGFP-fused PX domains from indicated proteins and subjected to live-cell imaging. The coverslip was mounted in the chamber with serum-free medium and epifluorescence images were taken every 15 sec. Images of before and after incubation with $100 \mathrm{nM}$ wortmannin for $10 \mathrm{~min}$ at room temperature are shown. Scale bars, $10 \mu \mathrm{m}$.

Fig. 5. Alignment of the amino acid sequences of the PX domains. The sequences were aligned using ClustalW2 and a Gonnet-weight matrix with a gap-opening penalty of 5 and extension penalty of 0.05 for multiple alignments with some modification by hand to adjust the positions of proline-rich motifs. The regions containing the strongly conserved (R/K)-(R/K)-(Y/F) xxFxxLxxxL and PxxP motifs are boxed, where ' $\mathrm{x}$ ' indicates any amino acid. The basic residues in the second anion-binding pocket of $\mathrm{p} 47^{\text {phox }}$ which directly interact with the polar head of PA are indicated in bold. The basic residues of RPK118 and KIF16B corresponding to those for PA binding of p47phox and those in the loop forming the second anion-binding pocket are indicated in bold and the residues mutated in this study are underlined. All PX domains in the alignment are from human homologs and the GeneBank accession numbers and corresponding amino acids are: RPK118, NP036556, 9-128; KIF16B, NP078980, 1184-1307; PLD1b, NP001123553, 79-209; p47phox NP000256, 4-121; p40 phox, NP000622, 18-136; SNX19, NP055573, 532-659.

Fig. 6. Subcellular localization of PX domain mutants incapable of binding to PI(3)P. Images of MDCK cells expressing EGFP-fused PX domains were taken as in Fig. 4. without any treatment of the cells. Scale bars, $10 \mu \mathrm{m}$.

Fig. 7. Effects of point mutations of the PX domain from RPK118 on lipid binding and subcellular localization. Protein-lipid overlay assay and fluorescent microscopy were conducted as in Figs. 1 and 4, using several point mutants of GST-fused or EGFP-fused PX domains from RPK118. Scale bars, $10 \mu \mathrm{m}$. 
Fig. 8. Co-localization of Y54A mutant of the PX domain from RPK118 with PLD on endosomes. A: Live-cell imaging was performed as in Fig. 4. Images of before and after treatment of the cells with $100 \mathrm{nM}$ PMA, and further treatment with $100 \mathrm{nM}$ wortmannin for $10 \mathrm{~min}$ at room temperature are presented. Scale bars, $10 \mu \mathrm{m}$. B: Immunofluorescence was performed as in Fig. 3, except that the cells were treated with $100 \mathrm{nM}$ PMA for $10 \mathrm{~min}$ at room temperature. C: COS7 cells cultured on coverslips were transiently transfected to co-express EGFP-RPKPX-Y54A and HA-tagged PLD1b. The cells were fixed after treatment with $100 \mathrm{nM}$ PMA for $10 \mathrm{~min}$ followed by immunofluorescence as in B, except that HA-PLD1b was visualized in red using mouse antibody against HA-tag instead of EEA1. 
Fig. 1 Takeuchi et al

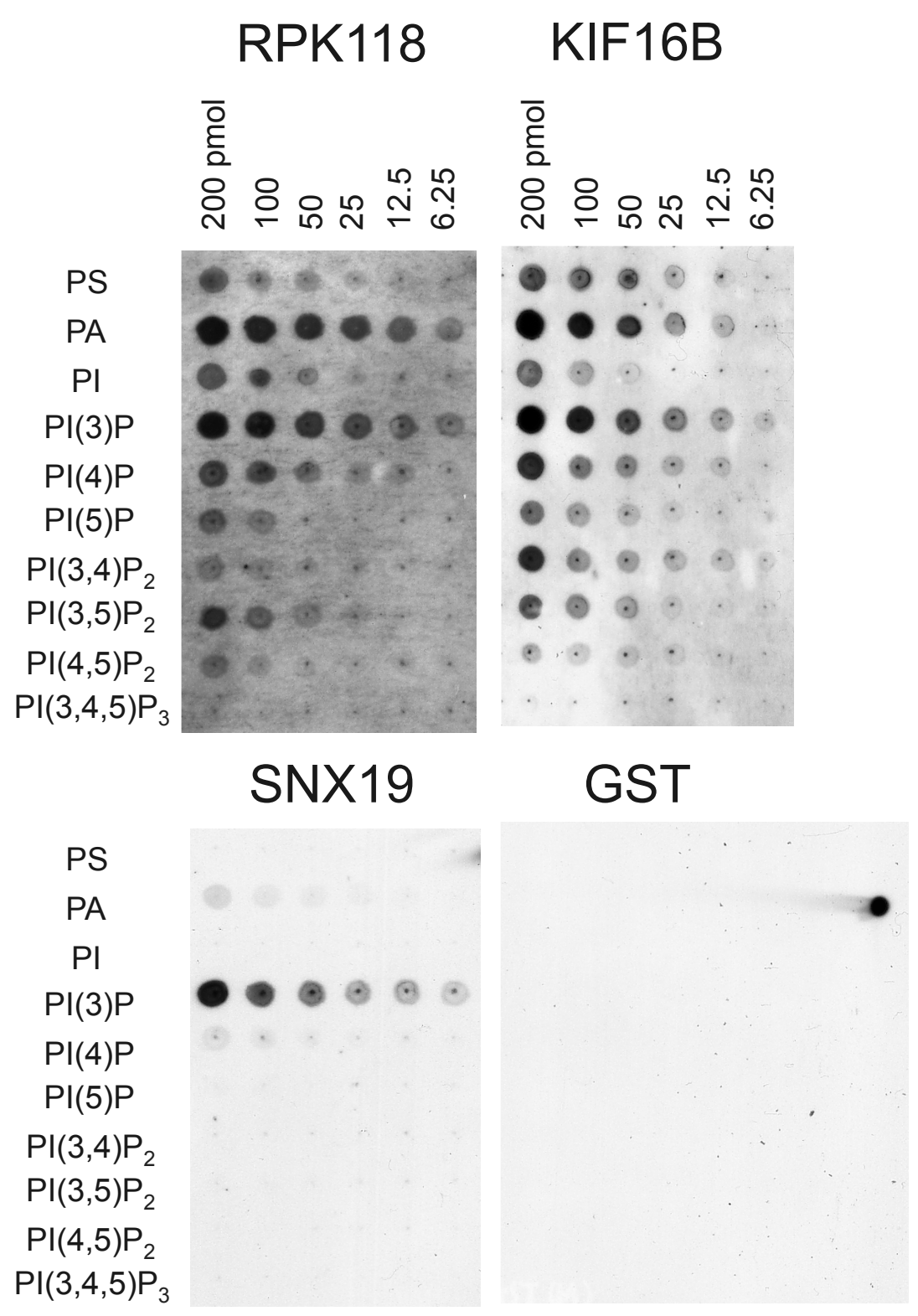


Fig. 2 Takeuchi et al

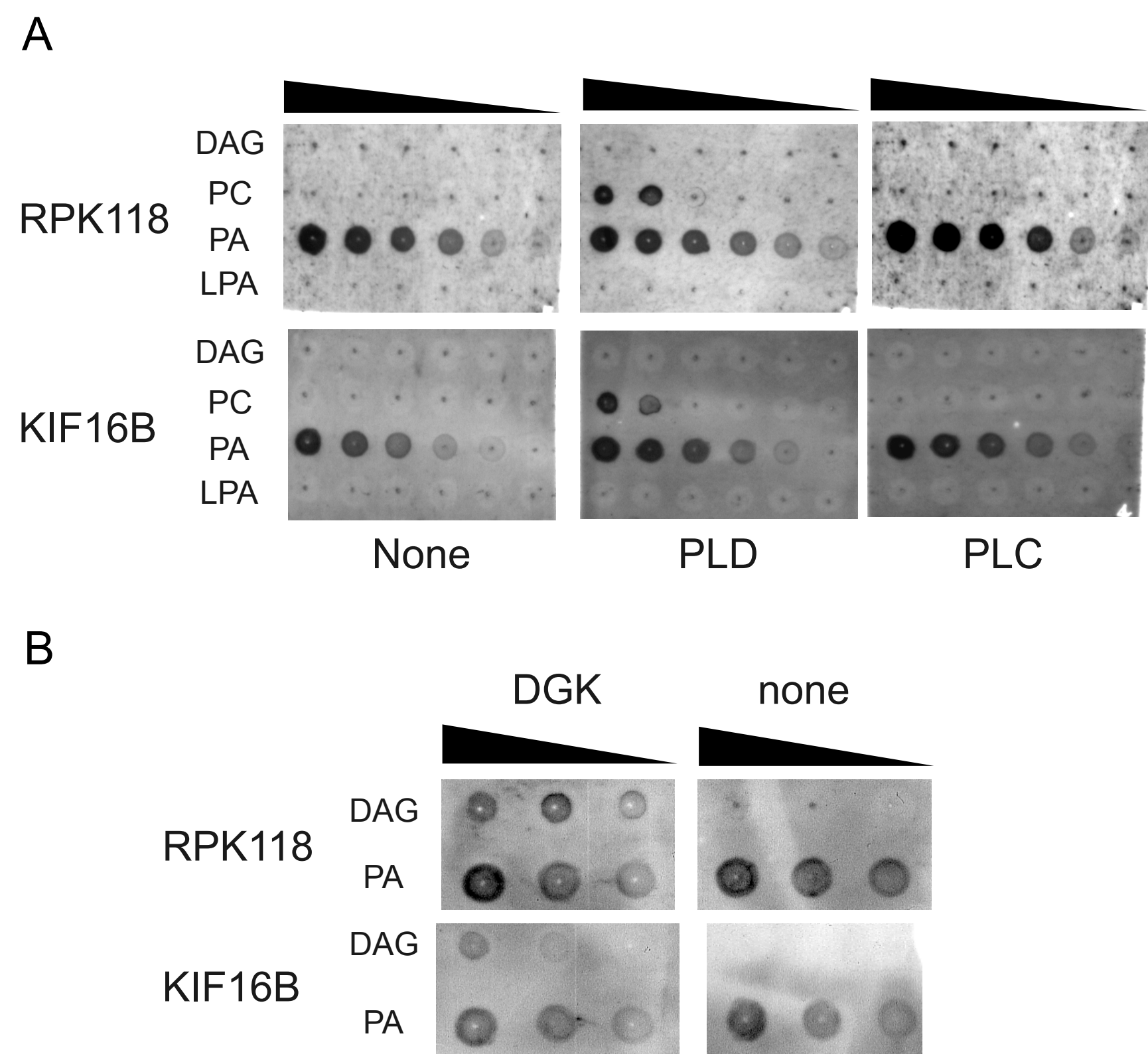


Fig. 3 Takeuchi et al

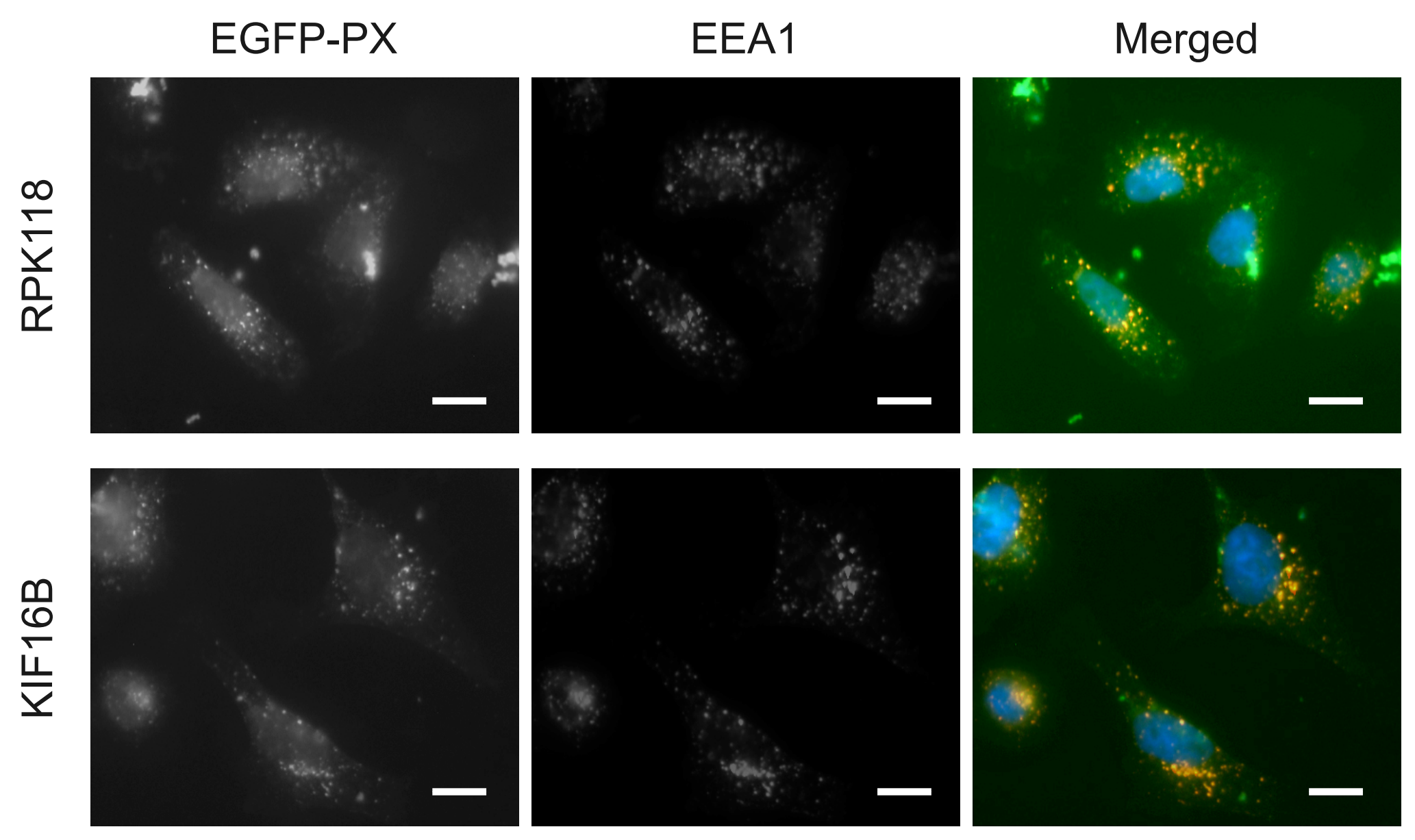


Fig. 4 Takeuchi et al

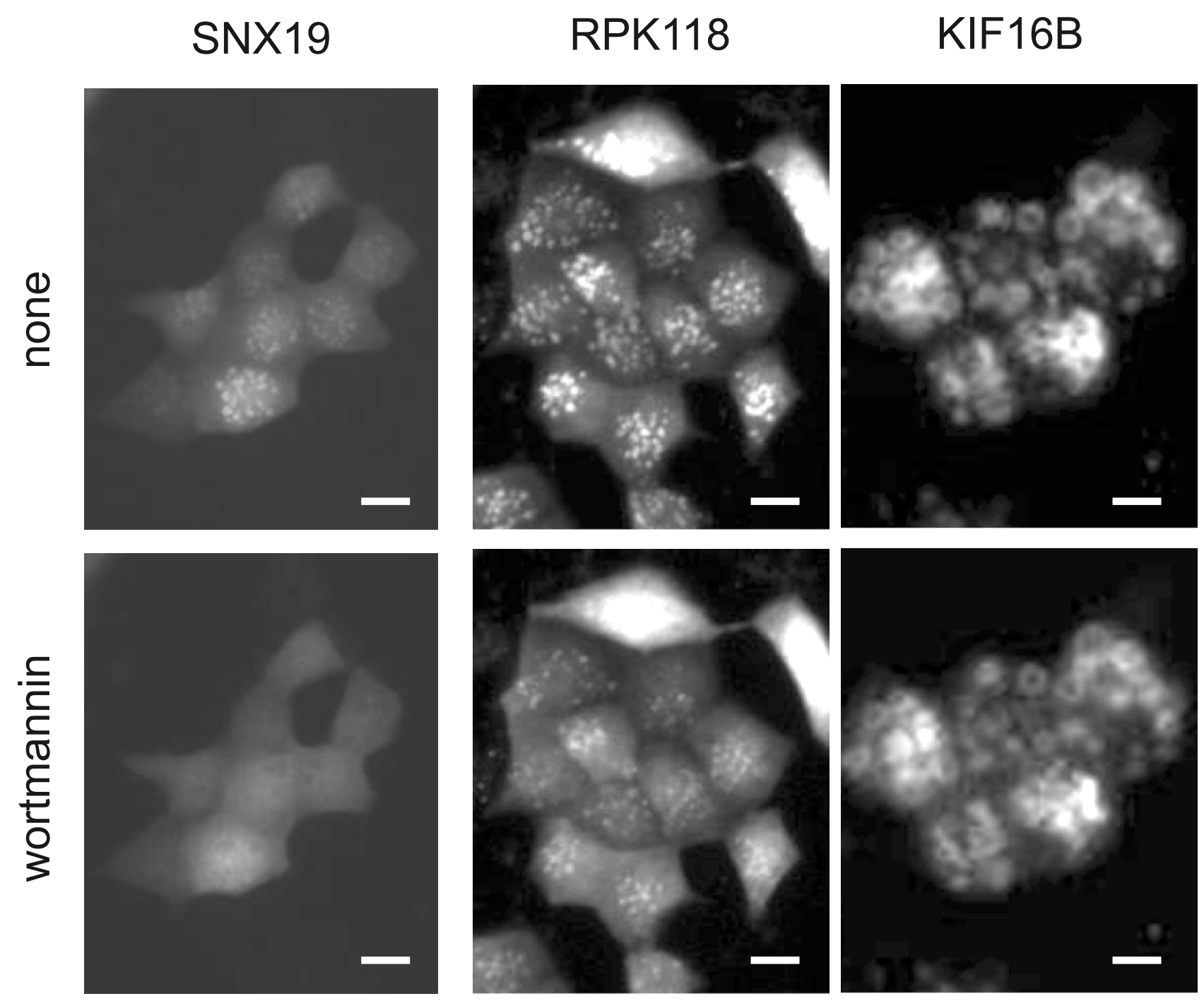




\section{Fig. 5 Takeuchi et al}

RPK118

KIF16B

PLD1

p4 7phox

p40phox

SNX19

RPK 118

KIF16B

PLD1

p4 7phox

p40phox

SNX19
9 ---ADLARFYTVTEPQRHPRGYTVYKVTAGVVSRRNP----EDVQEIIVWKRYSDFKKLHKELWQIH-------------- 68

1184 ----IKISIPRYVLCGQGKDAHFEFEVKITV-----------LDETWTVFRRYSRFREMHKTLKLKYA------------- 1236

79 CPIKAQVLEVERFTSTTRVPSINLYTIELTHG-----------EFKWQVKRKFKHFQEFHRELLKYKAFIRIPIPTRRHTF 148

4 --TFIRHIALLGFEKRFVPSQHYVYMFLVKWQ----------DLSEKVVYRRFTEIYEFHKTLKEMFP------------- 59

18 -DDVAISANIADIEEKRGFTSHFVFVIEVKTK----------GGSKYLIYRRYRQFHALQSKLEERFG------------- 74

532 -NLRITGTITAREHSGTGFHPYTLYTVKYETALDGENSSGLQQLAYHTVNRRYREFLNLQTRLEEK----------PDLRK 601

69 --KNLFRHSELFPPFAKGIVFGRFDETVIEERRQCAEDLLQFSAN-----------------IPALYN-SKQLEDFFKGG 128 1237 -------ELAALEFP KKLFGNKDERVIAERRSHLEKYLRDFFSVMLQSATSPLHINKVGLTLSKH-TICEFSPFFKKG 1307

149 RRQNVREEPREMPSLPRSSENMI-REEQFLGRRKQLEDYLTKILK-----------------MPMYRN-YHATTEFLDIS 209

60 IEAGINPENRI IPHLPAPKWF-DGQRAAENRQGTLTEYCSTLMS-----------------LPTKISRCPHLLDFFKVR 121

75 PDSKSSALACTIPTLPAKVYVG-VKQEIAEMRIPALNAYMKSLLS----------------LPVWVLMDEDVRIFFYQS 136

602 FIKNVKGPKKLFPDLPFGNM----DSDRVEARKSLLESFLKQLCA-----------------IPEIAN-SEEVQEFLALN 659 
Fig. 6 Takeuchi et al
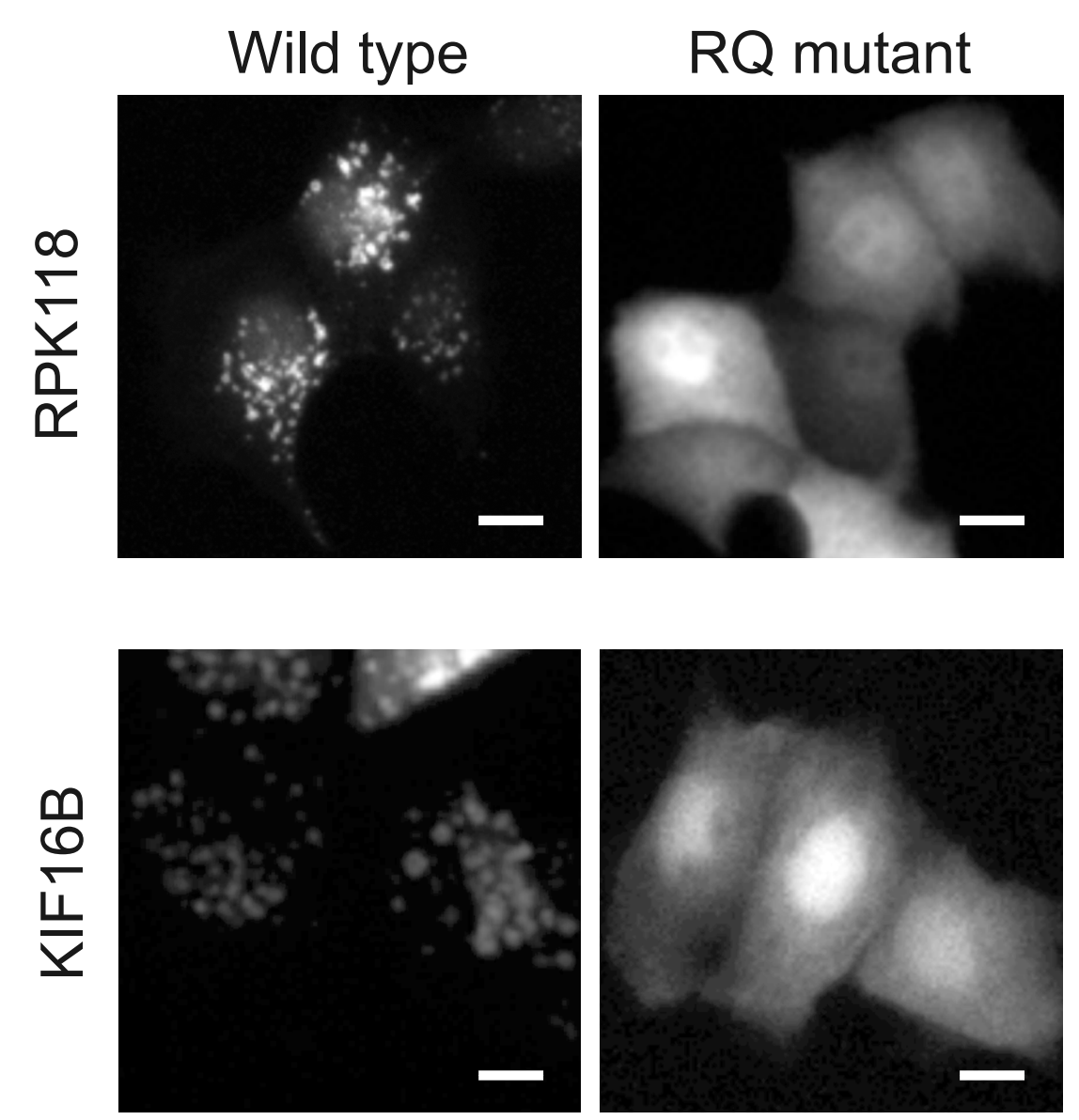
Fig. 7 Takeuchi et al

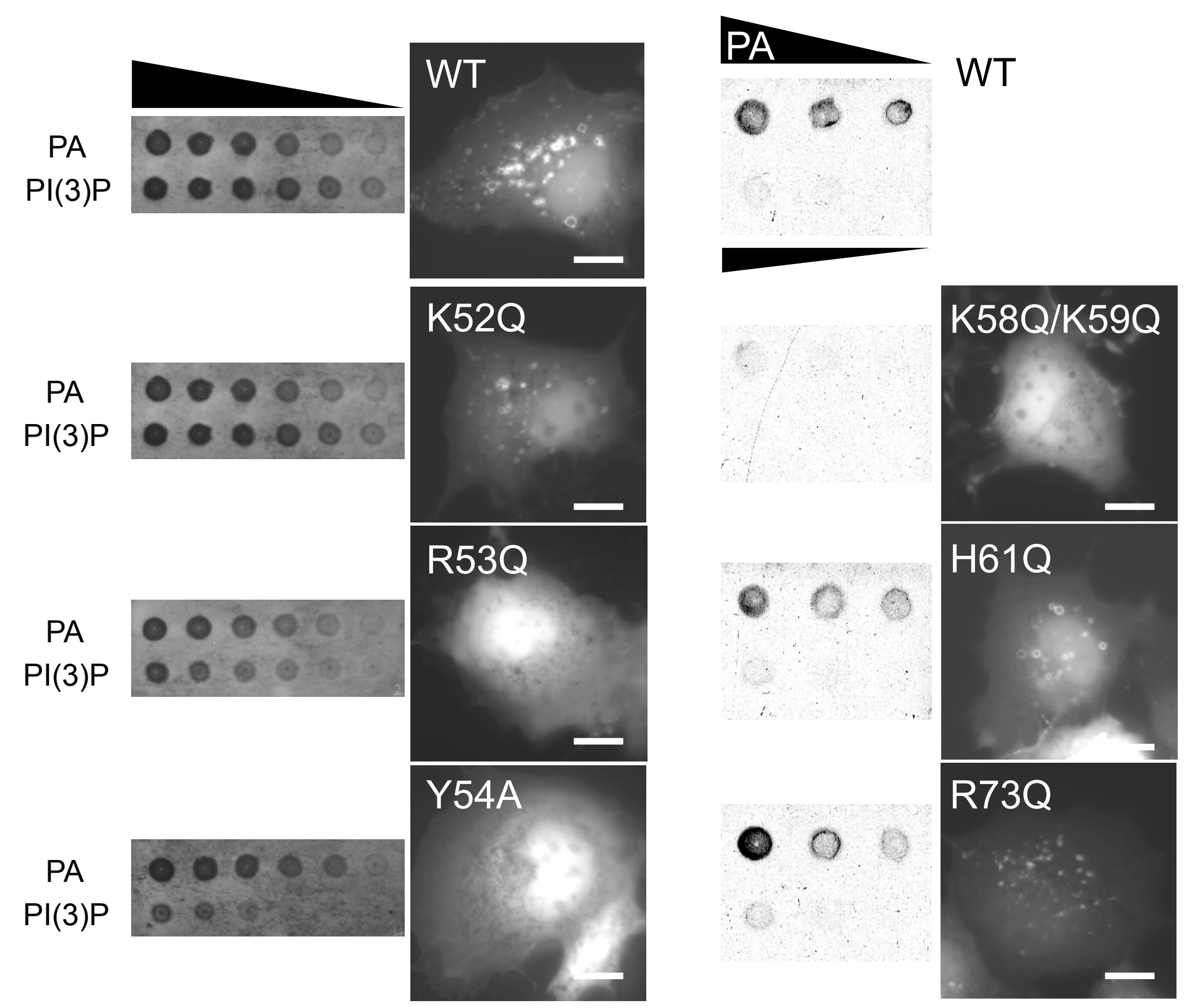


Fig. 8 Takeuchi et al

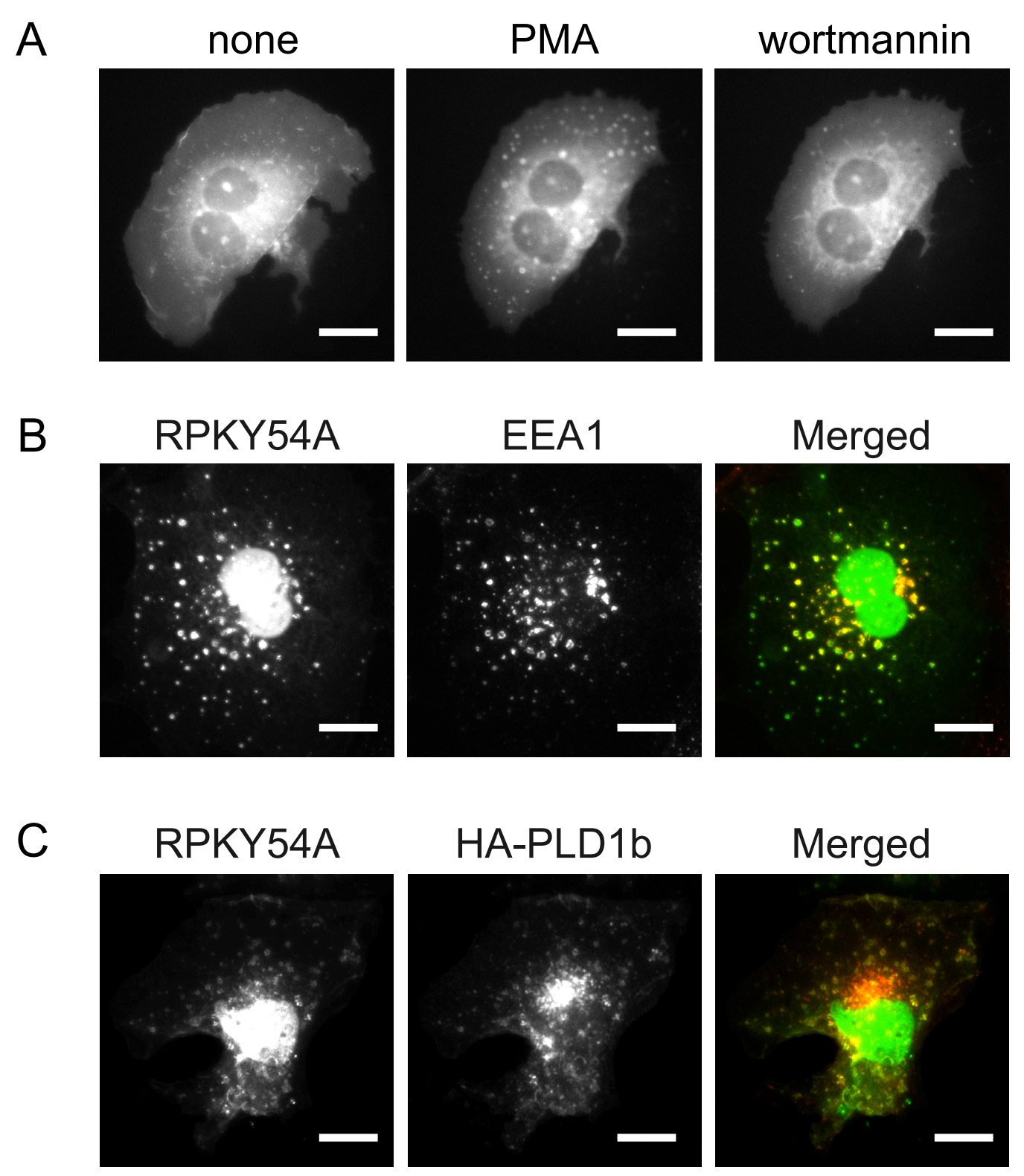

